\title{
Finite Element Model to Study Effect of Buffers Along With Leak from ER on Cytosolic $\mathrm{Ca}^{2+}$ Distribution in Oocyte
}

\author{
Sunil Panday ${ }^{1}$, Kamal Raj Pardasani ${ }^{2}$ \\ 1,2 (Department of Mathematics, Maulana Azad National Institute of Technology, Bhopal, India-462051)
}

\begin{abstract}
Calcium dynamics in oocytes plays an important role in oocyte maturation. The calcium concentration is regulated at high levels in oocytes through various mechanisms in order to meet the requirements of oocyte maturation. The understanding of these mechanisms are crucial in understanding the processes of reproduction. In this paper an attempt has been made to develop a finite element model of calcium dynamics in oocyte. The model incorporates the parameters like diffusion coefficient, leak from Endoplasmic Reticulum(ER), and buffers namely 1,2-bis(o-aminophenoxy)ethane- $N, N, N^{\prime}, N^{\prime}$-tetraacetic acid(BAPTA) and

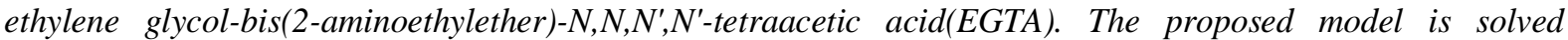
numerically using appropriate initial and boundary conditions. A program has been developed in MATLAB 7.11 for the entire problem and simulated on a 32-bit machine to compute the numerical results. The effect of BAPTA, EGTA and Leak from ER is studied in the neighbourhood of L-type calcium channel on calcium distribution in oocyte.
\end{abstract}

Keywords: Finite Element Method, EGTA, BAPTA, ER Leak, Reaction Diffusion Equation.

\section{INTRODUCTION}

In both plants and animals, free calcium is used as a second messenger in cell signaling that mediates a wide variety of biological processes. Almost all cells respond to oscillations of the free cytosolic calcium concentration to a variety of physical and chemical stimuli. Calcium wave and oscillations arise due to influx of $\mathrm{Ca}^{2+}$ through the plasma membrane Voltage Gated calcium channel, leak through ER membrane, influx through Inositol Triphosphate Receptor(IPR) channels on the ER membrane[1,2]. The calcium waves and oscillations also arise due to reuptake of $\mathrm{Ca}^{2+}$ from the cytosol into the ER by Sarco Endoplasmic Reticulum $\mathrm{Ca}^{2+}$ ATPase(SERCA) pump, into the extracellular space by Plasma Membrane $\mathrm{Ca}^{2+}$ ATPase (PMCA) pump and with the help of buffering by endogenous calcium buffers. The development of calcium imaging has allowed the spatiotemporal study of the global and elementary calcium release in cellular compartments of muscle cells, endothelial cells and egg cells[3,4]. Elementary calcium release events involving release through IPR channels in Xenopus oocytes, HeLa cells and analogous calcium sparks in cardiac, smooth and skeletal muscle are mediated through Ryanodine Receptor(RyR) channels. Most notably, the opening of L-type calcium channels is mediated by elevations of cytosolic calcium. This positive feedback underlies the process of calcium-induced calcium release (CICR), which accounts for the generation of propagating calcium waves[5,6].

Calcium concentration is strongly buffered in living cells[7,8]. Although the majority of buffers are relatively stationary, Zhou and Neher[9] have estimated that as much as $25 \%$ of cytoplasmic buffers in chromaffin cells are mobile. The time constant for buffering has been estimated to be in millisecond range or smaller, so that locally in space the concentration of free $\mathrm{Ca}^{2+}$, is determined by the association-dissociation equilibrium with the buffers[10]. The various characteristics of available $\mathrm{Ca}^{2+}$ buffer have been used successfully to study the kinetic properties and location of $\mathrm{Ca}^{2+}$-triggered events[11,12]. Several recent modeling studies have addressed the question of the effect of buffer properties on $\mathrm{Ca}^{2+}$ transients[13,14,15]. Sala and Hernandez-Cruz[16] calculated the amplitude and shape of $\mathrm{Ca}^{2+}$ transients resulting from voltage-gated $\mathrm{Ca}^{2+}$ entry in frog sympathetic ganglia neurons for a number of different buffer conditions, such as buffer diffusion rates, affinity, and kinetics[16]. The issue of how buffers affect the movement of $\mathrm{Ca}^{2+}$ in cells has recently arisen in the context of $\mathrm{Ca}^{2+}$ signaling from internal stores. Inositol 1,4,5-triphosphate produced by agonist-mediated receptors in the plasma membrane, is a potent effector of $\mathrm{Ca}^{2+}$ release from the ER[17]. This mechanism is now widely believed to be a key feature of $\mathrm{Ca}^{2+}$ oscillations in a number of cell types, including Hamster and Xenopus oocytes[5,18], RBL cells[19] and other cell types[17]. To understand these phenomena, knowledge of the effect of endogenous $\mathrm{Ca}^{2+}$ buffers, as well as exogenous buffers and indicators, which are important tools in studying $\mathrm{Ca}^{2+}$ release, is essential[10]. Oocyte maturation provides an exceptional model system to elucidate the mechanisms regulating $\mathrm{Ca}^{2+}$ signaling differentiation during cellular development, because $\mathrm{Ca}^{2+}$ signaling differentiation during oocyte maturation is essential for the egg to acquire developmental competence at fertilization[20,21,22]. In fact intracellular $\mathrm{Ca}^{2+}$ is the universal signal for egg activation in all sexually reproducing species[20,23], and the fertilization-induced specialized $\mathrm{Ca}^{2+}$ signal takes the form of a single, or multiple $\mathrm{Ca}^{2+}$ transients depending on the species[20,21]. Therefore, $\mathrm{Ca}^{2+}$ signals differentiate in a 
dramatic fashion during oocyte maturation to endow the egg with the capacity to respond to sperm and initiate development. A number of experimental and theoretical investigations on calcium dynamics in various cells like Neurons[24,25], Astrocytes[26], Fibroblasts cell[27] etc are reported in the literature. Very few attempts are reported for theoretical study of calcium dynamics in oocytes[2,10,21] but they have not studied the effect of Ltype calcium channels. Some experimental studies of calcium dynamics in oocytes have been reported in the literature[28,29,30] and have suggested that L-type calcium channels plays a significant role in oocyte activation. In view of above, an attempt has been made in this paper to study calcium dynamics in the neighbourhood of L-type calcium channels in oocyte. Finite element model of calcium distribution in oocyte has been developed for a one dimensional unsteady state case. The model has been used to study the effect of different buffers, leak from ER and L-type calcium channel on $\mathrm{Ca}^{2+}$ distribution in oocytes.

\section{MATHEMATICAL MODEL}

Intracellular $\mathrm{Ca}^{2+}$ kinetics in Oocyte is determined by a system of reaction-diffusion equation. Calcium buffering is governed by the bimolecular reaction

$$
\left[\mathrm{Ca}^{2+}\right]+\left[B_{j}\right] \underset{k^{-}}{\stackrel{k^{+}}{\rightleftarrows}}\left[\mathrm{CaB}{ }_{j}\right]
$$

where $\left[\mathrm{Ca}^{2+}\right],\left[\mathrm{B}_{j}\right]$ and $\left[\mathrm{CaB}_{j}\right]$ represent the cytosolic $\mathrm{Ca}^{2+}$ concentration, free buffer concentration and calcium bound buffer concentration respectively, and ' $j$ ' is an index over buffer species; $k_{j}^{+}$and $k_{j}^{-}$are on and off rates for $j^{\text {th }}$ buffer respectively. Using Fikian diffusion, the buffered reaction diffusion system in one-dimension is expressed as[14,31].

$$
\begin{gathered}
\frac{\partial\left[\mathrm{Ca}^{2+}\right]}{\partial t}=D_{C a} \nabla^{2}\left[\mathrm{Ca}^{2+}\right]+\sum_{j} R_{j}+\sigma_{C a} \\
\frac{\partial\left[B_{j}\right]}{\partial t}=D_{B_{j}} \nabla^{2}\left[B_{j}\right]+R_{j} \\
\frac{\partial\left[C a B_{j}\right]}{\partial t}=D_{C a B_{j}} \nabla^{2}\left[C a B_{j}\right]-R_{j}
\end{gathered}
$$

Where

$$
R_{j}=-k_{j}^{+}\left[B_{j}\right]\left[C a^{2+}\right]+k_{j}^{-}\left[C a B_{j}\right]
$$

$D_{C a}, D_{B j}, D_{C a B j}$ are diffusion coefficients of free calcium, free buffer and $C a^{2+}$ bound buffer respectively and $\sigma_{C a}$ is net influx of $\mathrm{Ca}^{2+}$ from the voltage gated calcium channel. Let $\left[B_{j}\right]_{T}=\left(\left[B_{j}\right]+\left[C a B_{j}\right]\right)$ be the total buffer concentration of $j^{\text {th }}$ buffer and the diffusion coefficient of buffer is not affected by the binding of calcium i.e. $D_{B j}=D_{C a B j}$. Then equation (5) can be written as[32]

$$
R_{j}=-k_{j}^{+}\left[B_{j}\right]\left[C a^{2+}\right]+k_{j}^{-}\left(\left[B_{j}\right]_{T}-\left[B_{j}\right]\right)
$$

By assuming buffer concentration is present in excess inside the cytosol so that the concentration of free buffer is constant in space and time, i.e. $\left[B_{j}\right] \cong\left[B_{j}\right]_{\infty}$. Under this assumption equation (6) is approximated by [33]

$$
k_{j}^{+}\left[B_{j}\right]\left[C a^{2+}\right] \approx k_{j}^{-}\left(\left[B_{j}\right]_{T}-\left[B_{j}\right]_{\infty}\right)
$$

where $\left[B_{j}\right]_{\infty}=k_{j}^{-}\left[B_{j}\right]_{T} /\left(k_{j}^{-}+k_{j}^{+}\left[\mathrm{Ca}^{2+}\right]_{\infty}\right)$ is the background buffer concentration. Thus for single mobile buffer species equation (2) can be written as $[32,33]$

$$
\frac{\partial\left[\mathrm{Ca}^{2+}\right]}{\partial t}=D_{C a} \nabla^{2}\left[\mathrm{Ca}^{2+}\right]-k_{j}^{+}\left[B_{j}\right]_{\infty}\left(\left[\mathrm{Ca}^{2+}\right]-\left[\mathrm{Ca}^{2+}\right]_{\infty}\right)+\sigma_{C a}
$$

Here $\left[\mathrm{Ca}^{2+}\right]$ is background calcium concentration. We assume a single point source of $\mathrm{Ca}^{2+}, \sigma_{\mathrm{Ca}}$ at $x=0$; there are no sources for buffers and buffer concentration is in equilibrium with $C a^{2+}$ far from the source. From GHK current equation [34], we have

$$
I_{C a}=P_{C a} z_{C a}^{2} \frac{F^{2} V_{m}}{R T}\left[\frac{C a_{i}-C a_{o} \exp \left(-z_{C a} F V_{m} /(R T)\right)}{1-\exp \left(-z_{C a} F V_{m} /(R T)\right)}\right]
$$

where $I_{C a}$ is the current due to calcium gradient, $P_{C a}$ is the calcium permeability, $z_{C a}$ is the valency of calcium ion (i.e, +2$), V_{m}$ is the membrane potential, $F$ is the Faraday's constant, $R$ is the gas constant, $T$ is the absolute temperature, $C a_{i}$ and $C a_{o}$ are the intracellular and extracellular $C a^{2+}$ concentration respectively. The net influx, $\sigma_{C a}$, of $\mathrm{Ca}^{2+}$ ions flowing per second at the origin is [35],

$$
\sigma_{C a}=-I_{C a} /\left(z_{C a} F V_{c y t}\right)
$$


where $V_{c y t}$ is the volume of the cytosol in oocyte. In equation (10) there is a negative sign before $I_{C a}$ because inward current is taken to be negative. The continuous leak of $\mathrm{Ca}^{2+}$ from ER membrane plays a crucial role in mediating cytosolic calcium. The expression of leak is given by[21]

$$
J_{\text {leak }}=V_{\text {leak }}\left(\left[\mathrm{Ca}^{2+}\right]_{\text {er }}-\left[\mathrm{Ca}^{2+}\right]\right)
$$

where $V_{\text {leak }}$ is the leak conductance and $\left[\mathrm{Ca}^{2+}\right]_{e r}$ is the Endoplasmic Reticulum $\mathrm{Ca}^{2+}$ concentration. Incorporating leak term from equation (11) into the equation (8), we get

$$
\frac{\partial\left[\mathrm{Ca}^{2+}\right]}{\partial t}=D_{C a} \nabla^{2}\left[\mathrm{Ca}^{2+}\right]-k_{j}^{+}\left[B_{j}\right]_{\infty}\left(\left[\mathrm{Ca}^{2+}\right]-\left[\mathrm{Ca}^{2+}\right]_{\infty}\right)+V_{\text {leak }}\left(\left[\mathrm{Ca}^{2+}\right]_{e r}-\left[\mathrm{Ca}^{2+}\right]\right)+\sigma_{C a}
$$

The initial and boundary conditions are given by [31]

Initial Condition:

Boundary Conditions:

$$
\left[\mathrm{Ca}^{2+}\right]_{t=0}=0.1 \mu M
$$

$$
\begin{gathered}
\lim _{x \rightarrow 0}\left(-D_{C a} \frac{\partial\left[C a^{2+}\right]}{\partial x}\right)=\sigma_{C a} \\
\lim _{x \rightarrow \infty}\left[C a^{2+}\right]=0.1 \mu M
\end{gathered}
$$

As stated in equation (15), $\left[\mathrm{Ca}^{2+}\right]_{\infty}$ tends to the background concentration of $0.1 \mu M$ as $x \rightarrow \infty$ but the domain taken by us is not infinite but a finite one. Usually it is believed that intracellular $\left[\mathrm{Ca}^{2+}\right]$ attains its equilibrium around $10 \mu \mathrm{m}$ [7]. Thus, we have taken the distance required by intracellular $\left[\mathrm{Ca}^{2+}\right]$ to attain background concentration to be $10 \mu \mathrm{m}$ along $x$-direction. The discretized variational form of equations (12) can be written as:

$$
\begin{gathered}
I^{(e)}=\frac{1}{2} \int_{x_{i}}^{x_{j}}\left[\left(\frac{\partial u^{(e)}}{\partial x}\right)^{2}+\left(\frac{1}{\lambda^{2}}\left(\left(u^{(e)}\right)^{2}-2 u^{(e)} u_{\infty}\right)\right)+\left(\frac{1}{\xi^{2}}\left(\left(u^{(e)}\right)^{2}-2 u^{(e)} u_{e r}\right)\right)+\left(\frac{1}{D_{C a}} \frac{\partial\left(u^{(e)}\right)^{2}}{\partial t}\right)\right] d x \\
-\psi^{e} \sigma_{C a} u^{(e)} /\left.\left(2 D_{C a}\right)\right|_{x=0}
\end{gathered}
$$

where $\lambda=\sqrt{D_{C a} /\left(k_{j}^{+} B_{T}\right)}, \xi=\sqrt{D_{C a} /\left(V_{\text {leak }}\right)}$. Here, we have used ' $u$ ' in lieu of $C a^{2+}$ for our convenience, $e=1,2,3 \ldots . .50$. We assume the length of each element as $0.2 \mu m$. The term in the last expression of equation (16), $\psi^{(e)}=1$ for $e=1$ and $\psi^{(e)}=0$ for rest of the elements. Here $\psi^{(e)}=1$ implies the locations/elements in which source of calcium is present. The shape function of concentration variation within each element is defined by:

$$
\begin{gathered}
u^{(e)}=c_{1}^{(e)}+c_{2}^{(e)} x \\
u^{(e)}=p^{T} c^{(e)}
\end{gathered}
$$

where

$$
p^{T}=[1 \quad x]
$$

where

$$
c^{(e)^{T}}=\left[c_{1}^{(e)} \quad \mathrm{c}_{2}^{(e)}\right]
$$

Substituting nodal conditions in equation (18), we get

where

$$
\bar{u}^{(e)}=P^{(e)} * c^{(e)}
$$

From the equation (21), we have

$$
\bar{u}^{(e)}=\left[\begin{array}{l}
u_{i} \\
u_{j}
\end{array}\right] \text { and } \quad P^{(e)}=\left[\begin{array}{cc}
1 & \mathrm{x}_{i} \\
1 & \mathrm{x}_{j}
\end{array}\right]
$$

where

$$
c^{(e)}=R^{(e)} * \bar{u}^{(e)}
$$

Substituting $c^{(e)}$ from equation (23) in (18), we get

$$
R^{(e)}=P^{(e)^{-1}}
$$

Now the integral $I^{(e)}$ can be written in the form

$$
u^{(e)}=p^{T} R^{(e)} \bar{u}^{(e)}
$$

$$
I^{(e)}=I_{t}^{(e)}+I_{k}^{(e)}+I_{m}^{(e)}-I_{s}^{(e)}-\psi^{(e)} I_{z}^{(e)}
$$


where

$$
\begin{gathered}
I_{t}^{(e)}=\frac{1}{2} \frac{d}{d t} \int_{x_{i}}^{x_{j}}\left[\frac{\left(u^{(e)}\right)^{2}}{D_{C a}}\right] d x \\
I_{k}^{(e)}=\frac{1}{2} \int_{x_{i}}^{x_{j}}\left[\frac{\partial u^{(e)}}{\partial x}\right]^{2} d x \\
I_{m}^{(e)}=\frac{1}{2} \int_{x_{i}}^{x_{j}}\left[\left(u^{(e)}\right)^{2}\left(\frac{1}{\lambda^{2}}+\frac{1}{\xi^{2}}\right)\right] d x \\
I_{s}^{(e)}=\frac{1}{2} \int_{x_{i}}^{x_{j}}\left[2 u^{(e)}\left(\frac{u_{\infty}}{\lambda^{2}}+\frac{u_{e r}}{\xi^{2}}\right)\right] d x \\
I_{z}^{(e)}=\sigma_{C a} u^{(e)} /\left.\left(2 D_{C a}\right)\right|_{x=0}
\end{gathered}
$$

Now we extremize the integral $I$ w.r.t. each nodal calcium concentration $u_{i}$ as given below

where

$$
\frac{d I}{d \bar{u}}=\sum_{e=1}^{50} \bar{M}^{(e)} \frac{d I^{(e)}}{d \bar{u}^{(e)}} \bar{M}^{(e)^{T}}=0
$$

and

$$
\bar{M}^{(e)}=\left[\begin{array}{cc}
0 & 0 \\
\cdot & \cdot \\
1 & 0 \\
0 & 1 \\
\cdot & \cdot \\
0 & 0
\end{array}\right], \quad \bar{u}=\left[\begin{array}{c}
u_{1} \\
u_{2} \\
\cdot \\
\cdot \\
\cdot \\
u_{51}
\end{array}\right], \quad I^{(e)}=\sum_{e=1}^{50} I^{(e)}
$$

$$
\frac{d I^{(e)}}{d \bar{u}^{(e)}}=\frac{d}{d t} \frac{d I_{t}^{(e)}}{d \bar{u}^{(e)}}+\frac{d I_{k}^{(e)}}{d \bar{u}^{(e)}}+\frac{d I_{m}^{(e)}}{d \bar{u}^{(e)}}-\frac{d I_{s}^{(e)}}{d \bar{u}^{(e)}}-\psi^{(e)} \frac{d I_{z}^{(e)}}{d \bar{u}^{(e)}}
$$

This leads to a following system of linear differential equations.

$$
[M]_{51 \times 51}[\dot{\bar{u}}]_{51 \times 1}+[K]_{51 \times 51}[\bar{u}]_{51 \times 1}=[F]_{51 \times 1}
$$

Here, $[\bar{u}]=\left[\begin{array}{llllll}u_{1} & u_{2} & u_{3} & u_{4} & \cdots \ldots \ldots \ldots \ldots . . . . . . . . . . u_{51}\end{array}\right], M$ and $K$ are system matrices and $F$ is system vector. The CrankNicolson method is employed to solve the system of equations (35).The time step is taken by us is $0.001 \mathrm{sec}$. A computer program in MATLAB 7.11 is developed to find numerical solution to the entire problem. The numerical values of biophysical parameters used in the model are as stated in the Table I[2,21,32].

\section{TABLE-I}

\begin{tabular}{lll}
\hline Symbol & Parameter & Value \\
\hline$F$ & Faraday's Constant & 96487 Coulombs/Mole \\
$R$ & Gas Constant & 8.314 Joule/Kelvin Mole \\
$T$ & Absolute Temperature & $37^{\circ} \mathrm{C}$ \\
$P_{C a}$ & Calcium Permeability & $2.6 \times 10^{-9} \mathrm{~m} / \mathrm{sec}$ \\
$V_{m}$ & Membrane Potential & $-0.03 \mathrm{Volts}$ \\
$V_{\text {leak }}$ & Leak Conductance & $0.002 / \mathrm{sec}$ \\
$z_{C a}$ & Valency of Calcium & 2 \\
$D_{C a}$ & Diffusion Coefficient of $\mathrm{Ca}^{2+}$ & $250 \mu \mathrm{m} / \mathrm{sec}^{2}$ \\
{$\left[C a^{2+}\right]_{e r}$} & Ca ${ }^{2+}$ Concentration in ER & $600 \mu \mathrm{M}$ \\
$k_{j}^{+}(E G T A)$ & On rate for EGTA & $3 / \mu M-\mathrm{sec}$ \\
$k_{j}^{-}(E G T A)$ & Off rate for EGTA & $1 / \mathrm{sec}$ \\
$k_{j}^{+}(B A P T A)$ & On rate for BAPTA & $100 / \mu \mathrm{M}-\mathrm{sec}$ \\
$k_{j}^{-}(B A P T A)$ & Off rate for BAPTA & $10 / \mathrm{sec}$ \\
$V_{c y t}$ & Volume of oocyte cytosol & $5.84 \times 10^{-11} \mathrm{litre}$ \\
$\mathrm{Ca} a_{O}$ & Extracellular Ca ${ }^{2+}$ Concentration & $3000 \mu M$ \\
\hline
\end{tabular}




\section{RESULTS AND DISCUSSION}

In this section, the numerical results for calcium profile are shown in the form of figures explaining the relationships observed between the physiological parameters and calcium concentration.
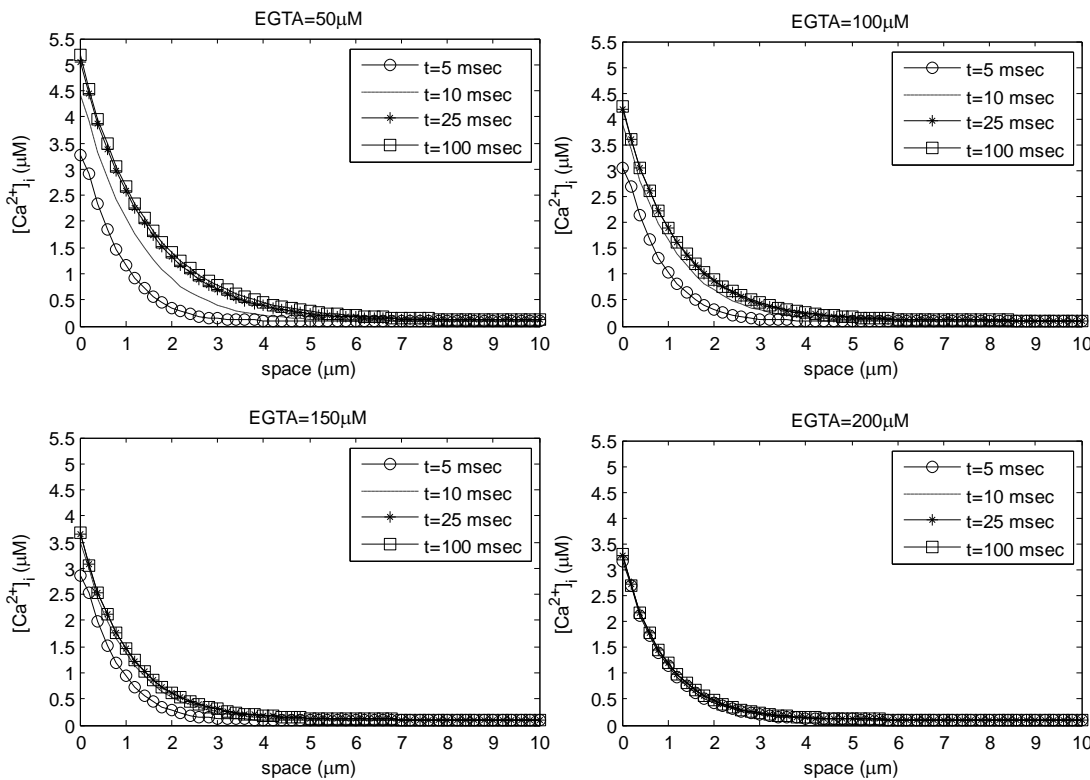

Fig. 1 Spatial $\left[\mathrm{Ca}^{2+}\right]_{\mathrm{i}}$ distribution for different instances of time and four different values of EGTA buffer Concentration.

Fig. 1 shows the intracellular $\mathrm{Ca}^{2+}$ ion distribution in space for different instances of time and for different values of EGTA buffer concentration. Here $\left[\mathrm{Ca}^{2+}\right]_{i}$ represents the intracellular calcium concentration. In fig. 1, we observe that as time elapses the cytosolic calcium concentration increases. For $t=5 \mathrm{msec}$, the calcium concentration attains background concentration within $3 \mu \mathrm{m}$. But as time elapses the level of $\mathrm{Ca}^{2+}$ concentration is increasing and background concentration is achieved beyond $6 \mu \mathrm{m}$. In fig. 1, we also observe that with increase in EGTA concentration, the cytosolic $\mathrm{Ca}^{2+}$ concentration decreases. For EGTA buffer concentration of $200 \mu M$ and $t=100 \mathrm{msec}$, the background $\mathrm{Ca}^{2+}$ concentration of $0.1 \mu M$ is achieved at $3 \mu M$ distance. But for EGTA buffer concentration of $50 \mu M$ and $\mathrm{t}=100 \mathrm{msec}$, the background $\mathrm{Ca}^{2+}$ concentration of $0.1 \mu M$ is achieved at a distance of $6 \mu \mathrm{m}$ from the source.
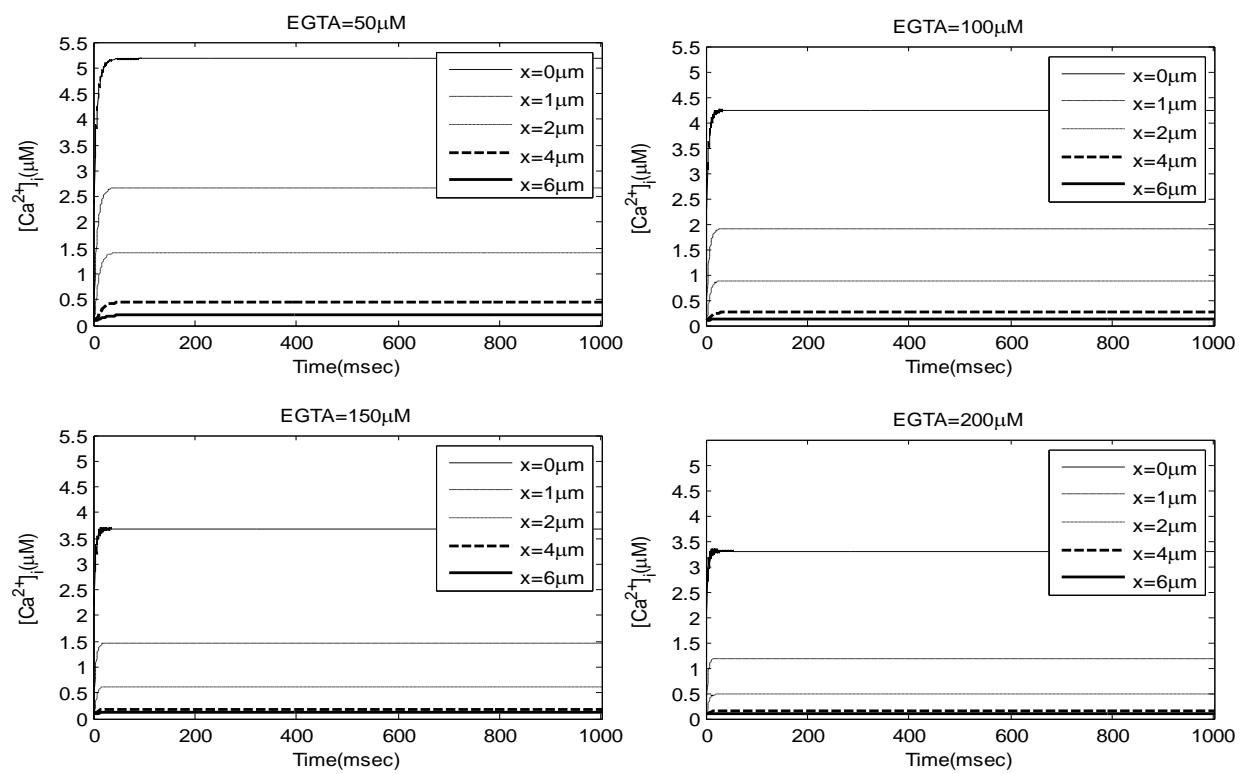

Fig. 2 Temporal $\left[\mathrm{Ca}^{2+}\right]_{\mathrm{i}}$ distribution at different nodal positions along $\mathrm{x}$-direction in oocytes for four different values of EGTA buffer concentration 


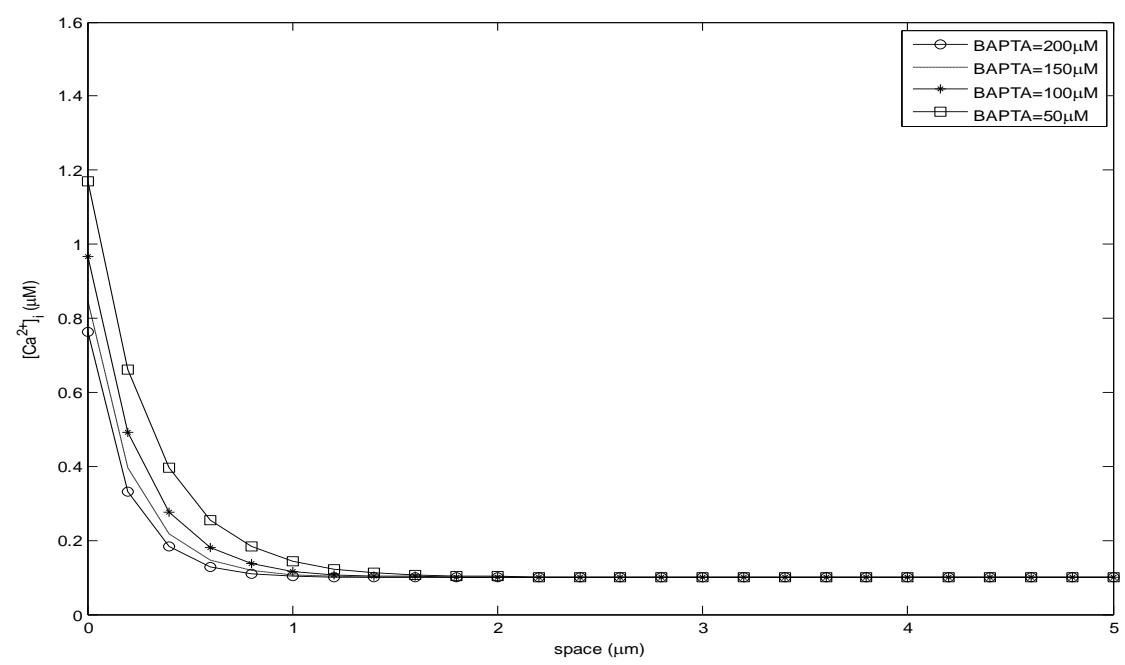

Fig. 3 Steady state spatial $\left[\mathrm{Ca}^{2+}\right]_{\mathrm{i}}$ distribution four different values of BAPTA buffer Concentration

Fig. 2 shows the temporal calcium distribution at different positions along $x$-direction in oocytes for four different values of EGTA buffer concentration. The effect of changing buffer concentration is more visible in fig 2 as compared to fig. 1. From fig. 2 it is observed that the peak value of $\mathrm{Ca}^{2+}$ concentration is higher for lower concentration of EGTA buffer. The effect of changing EGTA buffer concentration is observed within $5 \mu \mathrm{m}$ distance from the calcium channel. It is also observed that the steady state is achieved early for higher buffer concentration. This is because more calcium binds with buffers in case of higher concentration of buffers and thereby forcing the system to achieve steady state early.

Fig. 3 shows the steady state spatial variation in calcium concentration for different instances of time and four different values of BAPTA buffer concentration. In fig. 3, we observe that for higher concentration of BAPTA buffer, the $\mathrm{Ca}^{2+}$ concentration is lower. The results obtained here are similar to that in fig.1 but the fall in $\mathrm{Ca}^{2+}$ concentration in fig. 3 is quite sharp as compared to that in Fig 1. In fig. 3 calcium concentration achieve background concentration within $2 \mu \mathrm{m}$ distance from the calcium channel. This is because BAPTA is a fast buffer and thus binds $\mathrm{Ca}^{2+}$ very quickly in comparison to EGTA.
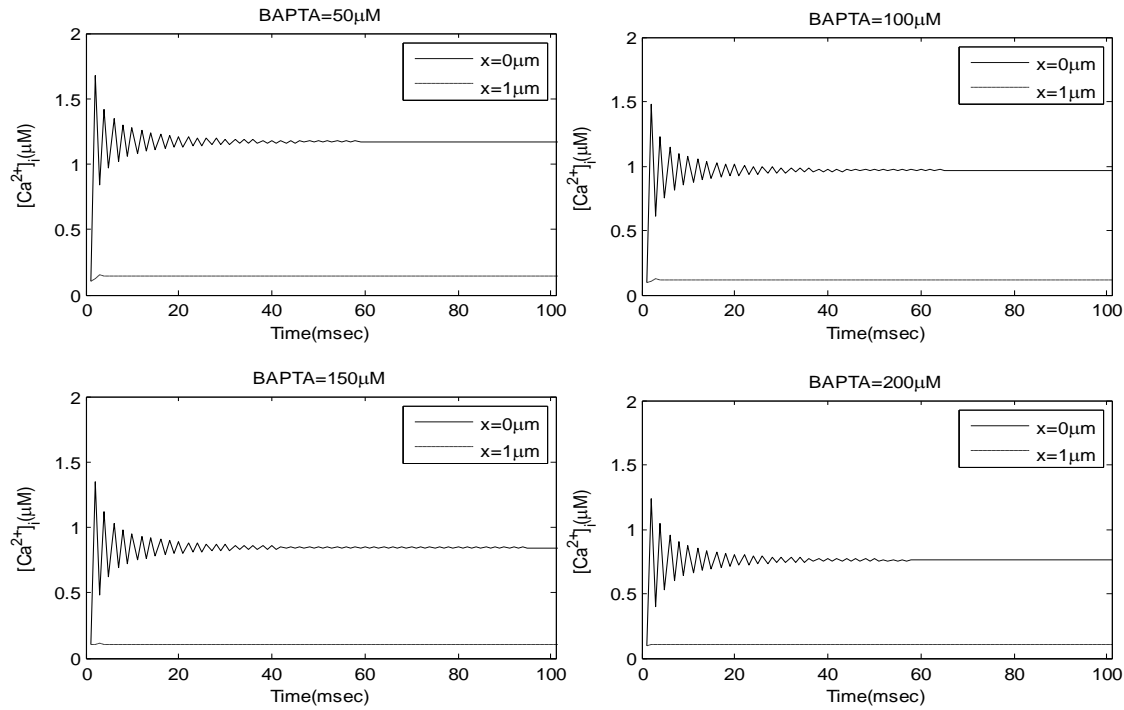

Fig. 4 Temporal $\left[\mathrm{Ca}^{2+}\right]_{i}$ at different nodal positions along $x$-direction in oocytes for four different values of BAPTA buffer concentration

Fig. 4 shows the intracellular temporal $\mathrm{Ca}^{2+}$ distribution at different positions along $x$-direction in oocytes for four different values of BAPTA buffer concentration. We observe in fig. 4 that the steady state peak value of calcium concentration in oocytes is higher for lower buffer concentration. Further, in fig. 4 we observe oscillations in calcium profiles in oocytes at the source location. This is because BAPTA buffers are fast buffers and they bind calcium very quickly and their capacity to bind calcium is higher than the influx. Thus this 
difference in binding capacity and influx seems to be the cause of oscillations initially in calcium profiles. These oscillations disappear after $50 \mathrm{msec}$ which imply that buffer gets saturated by binding enough calcium and therefore the calcium concentration in the cell near the source becomes constant in some proportion to influx.
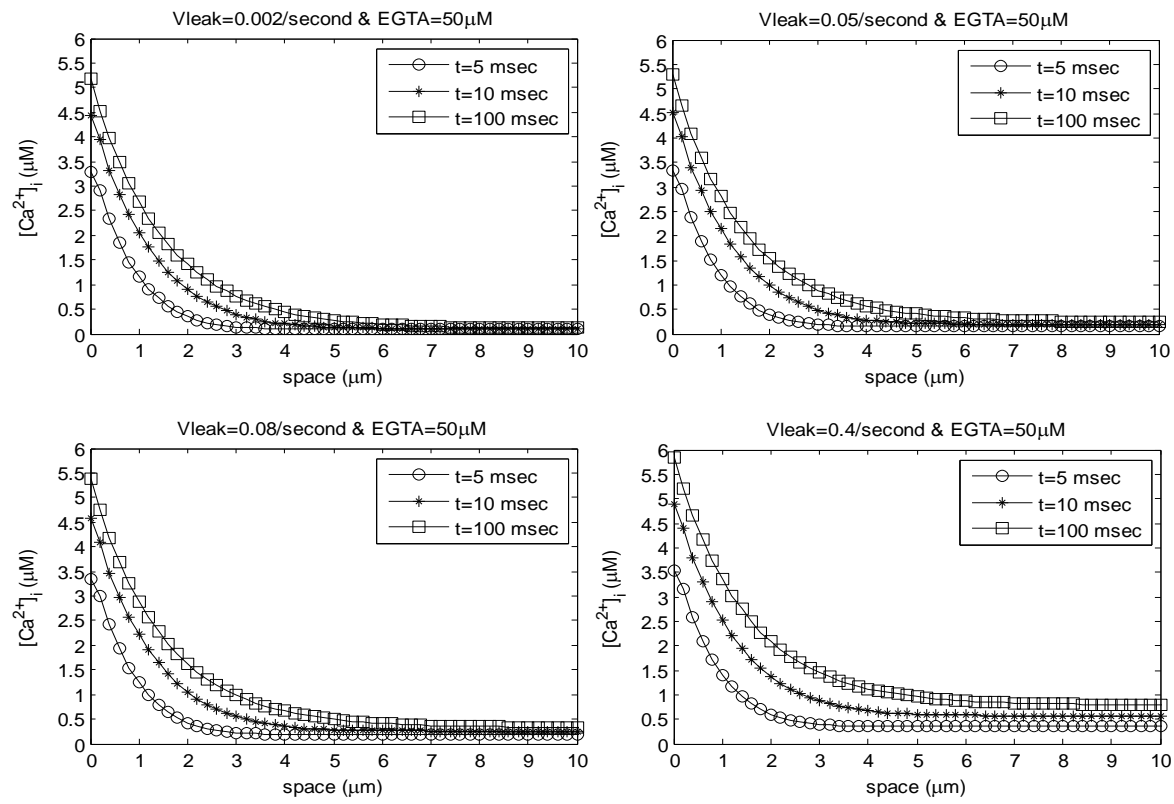

Fig. 5 Spatial $\left[\mathrm{Ca}^{2+}\right]_{\mathrm{i}}$ distribution for different instances of time and four different values of $V_{\text {leak }}$ in the presence of EGTA buffer

Fig. 5 shows the intracellular spatial $\mathrm{Ca}^{2+}$ distribution at different points of time in presence of EGTA buffer and four different values of ER leak coefficient; $V_{\text {leak }}=0.002 / \mathrm{sec}, V_{\text {leak }}=0.05 / \mathrm{sec}, V_{\text {leak }}=0.08 / \mathrm{sec}$ and $V_{\text {leak }}=0.4 / \mathrm{sec}$. In Fig 5, we observe that as we increase the value of $V_{\text {leak }}$ from $0.002 / \mathrm{sec}$ to $0.08 / \mathrm{sec}$, there is a slight change in distance from the source where it achieves background concentration. But when we take the value of $V_{\text {leak }}$ is $0.4 / \mathrm{sec}$, there is a sudden change in the level of free calcium ion concentration and it achieves background calcium concentration beyond $10 \mu \mathrm{m}$ from the source. This is because the influx of calcium concentration takes place through ER leak and upto some extent the influx is balanced by other processes in cytosol. But when influx through ER leak is higher beyond the capacity of EGTA buffer and other processes in cytosol to balance it, the calcium concentration in cytosol is raised significantly.

\section{CONCLUSION}

A finite element model has been developed to study effect of ER leak, BAPTA and EGTA buffers on calcium distribution in oocyte for a one dimensional unsteady state case. It is concluded that the BAPTA has significant effect on calcium distribution in oocyte near the channel between $x=0$ to $x=1 \mu \mathrm{m}$ and during $t=0$ to $\mathrm{t}=100 \mathrm{msec}$. The BAPTA buffer plays an important role in quickly reducing the free calcium concentration inside the cytosol in order to regulate free ion concentration to desired level upto a required distance from source within short period of time. But EGTA and ER leak has significant effect on calcium distribution in oocyte between $x=0 \mu \mathrm{m}$ to $10 \mu \mathrm{m}$. EGTA buffers being slow buffers allow to regulate little higher levels of calcium concentration than background calcium concentration upto wider distances from the source as compared to that in BAPTA buffers, ER leaks also play important role by increasing the free ion concentration in cytosol upto wider distances from the mouth by increasing influx from the leak. Such models can be developed to study the relationships of intracellular $\mathrm{Ca}^{2+}$ concentration with other biophysical parameters in oocyte to generate information which may be of great use to biomedical scientists in understanding the mechanisms of oocyte cell growth and maturation of oocyte and reproduction.

\section{Acknowledgements}

The authors are highly thankful to Council of Scientific and Industrial Research, New Delhi, India for providing financial assistance to carry out this work. 


\section{REFERENCES}

[1] Z. Machaty, J. J. Ramsoondar, A. J. Bonk, K. R. Bondioli and R. Prather, Capacitative Calcium Entry Mechanism in Porcine Oocytes, Biology of Reproduction 66(3) (2002) 667-674

[2] M. S. Jafri, S. Vajda, P. Pasik and B. Gillo, A membrane model for Cytosolic calcium oscillations. A study using Xenopus oocytes, Biophysical Journal 63 (1992) 235-246.

[3] J.G.Barbara, $\mathrm{IP}_{3}$-dependent calcium-induced calcium release mediates bidirectional calcium waves in neurons: functional implications for synaptic plasticity, Biochimica et Biophysica Acta 1600 (2002) 12-18.

[4] N. L. Allbritton and T. Meyer, Localized calcium spikes and propagating calcium waves, Cell Calcium, Elsevier 14(10) (1993) 691697.

[5] J. D. Lechleiter and D. E. Clapham, Molecular mechanisms of intracellular calcium excitability in X. laevis oocytes, Cell (69) (1992) 283-294.

[6] X. P. Sun, N. Callamaras, J. S. Marchant and I. Parker, A continuum of $\mathrm{InsP}_{3}$-mediated elementary $\mathrm{Ca}^{2+}$ signalling events in Xenopus oocytes, The Journal of Physiology 509(1) (1998) 67-80.

[7] N. L. Allbritton, T. Meyer and L. Stryer, Range of messenger action of calcium ion and inositol 1,4,5-triphosphate, Science 258 (1992) 1812-1815.

[8] R. E. Milner, K. S. Famulski and M. Michalak, Calcium binding proteins in the sacroplasmic/endoplasmic reticulum of muscle and nonmuscle cells, Molecular and Cellular Biochemistry 112 (1992) 1-13.

[9] Z. Zhou and Neher E, Mobile and immobile calcium buffers in bovine adrenal chromaffin cells, The Journal of Physiology 469 (1993) 245-273.

[10] J. Wagner and J. Keizer, Effects of Rapid Buffers on $\mathrm{Ca}^{2+}$ Diffusion and $\mathrm{Ca}^{2+}$ Oscillations, Biophysical Journal 67 (1994) 447-456.

[11] A. Marty and E. Neher, Potassium channels in cultured bovine adrenal chromaffin cells, The Journal of physiology 367 (1985) 117 141.

[12] E. M. Adler, G. J. Augustine, S. N. Duffy and M. P. Charlton, Alien intracellular calcium chelators attenuate neurotransmitter release at the squid giant synapse, Journal of Neuroscience 11 (1991) 1496-1507.

[13] J. A. Cannor and G. Nikolakopoulos, Calcium diffusion and buffering in nerve cytoplasm, Lectures on Mathematics in the Life Sciences 15 (1982) 79-101.

[14] E. Neher, Concentration profiles of intracellular $\mathrm{Ca}^{2+}$ in the presence of diffusible chelators, Experimental Brain Research 14 (1986) $80-96$.

[15] F. Sala and A. H. Cruz, Calcium diffusion modeling in a spherical neuron. Relevance of buffering properties, Biophysical Journal 57(2) (1990) 313-324

[16] M. C. Nowyckt and M. J. Pinter, Time courses of calcium and calcium-bound buffers following calcium influx in a model cell, Biophysical Journal 64 (1993) 77-91.

[17] M. J. Berridge, In Cell to Cell Signalling from experiments to Theoretical Models. A. Goldbeter, Academic Press, New York (1989) 449-459.

[18] S. Miyazaki, M. Yuzaki, K. Nakada, H. Shirakawa, S. Nakanishi, S. Nakade and K. Mikoshiba, Block of $\mathrm{Ca}^{2+}$ wave and $\mathrm{Ca}^{2+}$ oscillation by antibody to the inositol 1,4,5-triphosphate receptor in fertilized hamster eggs, Science 257 (1992) 251-255.

[19] T. Meyer and L. Stryer, Calcium spiking, Annual Review of Biophysics and Biophysical Chemistry 20 (1991) 153-174.

[20] S. A. Stricker, Comparative biology of calcium signalling during fertilization and egg activation in animals, Developmental Biology $211(2000)$ 157-176

[21] G. Ullah, P. Jung and K. Machaca, Modeling $\mathrm{Ca}^{2+}$ signaling differentiation During Oocyte Maturation, Cell Calcium, Elsevier 42 (2007) 556-564.

[22] S. T. Homa, J. Carroll and K. Swann, The role of calcium in mammalian oocyte maturation and egg activation, Human Reproduction 8 (1993) 1274-1281.

[23] A. F. Antoine, J. E. Faure, S. Cordeiro, C. Dumas, M. Rougier and J. A. Feijo, A calcium influx is triggered and propagates in the zygote as a wave front during in vitro fertilization of flowering plants, Proceedings of the National Academy of Sciences, U.S.A. 97 (2000) 10643-10648.

[24] S. Tiwari and K. R. Pardasani, Finite Element Model to study Two dimensional unsteady state cytosolic calcium diffusion in presence of Excess Buffers, IAENG International Journal of Applied Mathematics 40(3) (2010) 108-112.

[25] A. Tripath and N. Adlakha, Finite Element Model to Study Calcium Diffusion in Neuron Cell in Presence of Excess Buffer for One Dimensional Steady State Global Journal of Computational Science \& Mathematics 1(1) (2011) 21-30.

[26] B. K. Jha, N. Adlakha and M. N. Mehta, Analytic Solution of Two Dimensional Advection Diffusion Equation Arising In Cytosolic Calcium Concentration Distribution, International Mathematical Forum Hikari 7(3) (2012) 135-144.

[27] M. Kotwani, N. Adlakha and M. N. Adlakha Numerical Model to Study Calcium Diffusion in Fibroblasts Cell for One Dimensional Unsteady State Case, Applied Mathematical Sciences, Hikari 6(102) (2012) 5063--5072.

[28] M. Tomkowiak, P. Guerrier and S. Krantic, Meiosis reinitiation of mussel oocytes involves L-type voltage gated calcium channel, Journal of Cellular Biochemistry 64(1) (1997) 152-160.

[29] I. Neant, L. Dufresne, J. Morasse, C. Gicquaud, P. Guerrier and F. Dube, The release from metaphase arrest in blue mussel oocytes, The International Journal of Developmental Biology 38(3) (1994) 513-523.

[30] A. P. Lopez, M. C. Gould and J. L. Stephano, MAPK in involved in metaphase I arrest in oyster and mussel oocytes, Biology of the Cell 95 (2003) 275-282.

[31] G. D. Smith, Analytical Steady State Solution to the rapid buffering approximation near an open $\mathrm{Ca}^{2+}$ channel, Biophysical Journal 71 (1996) 3064-3072.

[32] S. Tiwari, A Variational-Ritz Approach to Study Cytosolic Calcium Diffusion in Neuron Cells for a One dimensional Unsteady State Case, GAMS Journal of Mathematics and Mathematical Biosciences 2 (2009) 1-10.

[33] G. D. Smith, L. Dai, R. M. Miura and A. Sherman, Asymptotic Analysis of Buffered $\mathrm{Ca}^{2+}$ Diffusion Near a Point Source, SIAM Journal of Applied Math 61 (2000) 1816-1838.

[34] J. Keener and J. Sneyd, Mathematical Physiology, Springer 8 (1998) 53-56.

[35] B. K. Jha, N. Adlakha and M. N. Mehta, Finite Volume Model to Study The effect of Voltage Gated Calcium Channel on cytosolic Calcium Advection Diffusion, World Academy of Science, Engineering and Technology 5(2) (2011) 73-77. 